\title{
A Classical Explanation for Relativistic Longitudinal and Transverse Mass Increase
}

\author{
Declan Traill \\ Melbourne, Australia \\ Email: declan@netspace.net.au
}

How to cite this paper: Traill, D. (2021) A Classical Explanation for Relativistic Longitudinal and Transverse Mass Increase. Journal of Applied Mathematics and Physics, 9, 1904-1910.

https://doi.org/10.4236/jamp.2021.98124

Received: June 27, 2021

Accepted: August 8, 2021

Published: August 11, 2021

Copyright (อ 2021 by author(s) and Scientific Research Publishing Inc. This work is licensed under the Creative Commons Attribution International License (CC BY 4.0).

http://creativecommons.org/licenses/by/4.0/ (c) (i) Open Access

\begin{abstract}
In this paper, I show that the Special Relativistic effect of mass increase that occurs within objects when they move at high speed can be explained using Classical Physics principles. In my analysis, the wave nature of condensed matter and energy/mass equivalence are taken into consideration. The wave nature of particles is modelled as the particle structure comprising three-dimensional standing waves. When this is done, the difference in the energy sum of the component waves that comprise a particle moving at a high speed, when compared to those of a stationary particle, completely accounts for the mass increase. Furthermore, the additional momentum of the wave components in the direction of motion (the Longitudinal direction) is the cause of the inertia, or effective mass, of the object being greater than that in the Transverse direction (orthogonal to the direction of motion).
\end{abstract}

\section{Keywords}

Special, Relativity, Classical, Physics, Relativistic, Mass, Longitudinal, Transverse, Inertia, Component, Increase, Standing Wave, Equivalence, Condensed, Matter

\section{Introduction}

Relativists often object to the suggestion that a fast-moving object's mass increases (even though it was Einstein's Relativity that originally introduced the concept, however, later on the backed-away from the idea), stating that the mass of any particle is invariant and only the energy and momentum of the particle increase when it moves fast. However, their objection relates to the rest-mass of the particle, the particle is not at rest, it is moving at high speed. So, the objection is really not relevant. The particle attains additional energy when in high-speed motion and this energy has a mass equivalent which is the particle's 
Gravitational mass and, in the case of the Transverse direction, its inertial mass too.

The key to understanding Relativistic mass increase is the understanding that solid matter particles are actually comprised of three-dimensional standing waves [1]. When a stationary particle is accelerated to a high speed, the wave function that comprises the particle can be thought of as being the sum of an "upstream" and a "downstream" wave relative to the particle's direction of motion through space. Each of the two waves (upstream and downstream) that comprise a particle has a certain energy associated with it (depending on that wave's frequency) and the total energy of the particle is the sum of the energies of the "upstream" and "downstream" waves. Once this total energy has been calculated, then the mass equivalent for that energy can be calculated using the famous equation:

$$
E=m c^{2}
$$

The three-dimensional standing waves that form particles are defined by wave functions (solutions to the Classical and Schrödinger wave equations) [1] that describe how their component waves evolve in Space-Time. However, to simplify the calculations we can use the standing wave inside a laser's resonating cavity as a model for a particle, as it is comprised of an "upstream" and "downstream" wave that are summed, resulting in a standing wave. The laser is modeled here as an ideal laser with no energy loss and perfect reflection at the mirrors. The following analysis shows how the Relativistic mass increase can be explained from such a model.

The significance of this modeling is that the mass increase (which is different in the longitudinal and transverse directions) can be fully modeled and explained using Classical Physics, with no reference to Relativity required. The same Lorentz factor used in Relativity is used here too (I refer to dilated time and contracted length, both of which result from the Lorentz Transformations), but this had its origins prior to the emergence of Relativity and can be fully explained classically in the analysis of the light-clock thought experiment often used to explain Relativity. Furthermore, this modeling gives additional credibility to the modeling of fundamental particles as three-dimensional Electromagnetic standing waves, again using only Classical Physics. Lorentz outlined a similar Classical explanation for the change in inertial mass based on Electromagnetic considerations (although not with particles modeled as spherical standing waves) in his work [2].

\section{Classical Explanation for Relativistic Mass Increase}

The proof that this approach can work mathematically follows:

For a stationary laser/particle, let:

$e_{0}=$ The energy of the wave per unit length of the upstream/downstream wave.

$L_{0}=$ The length of the laser's resonant cavity (or particle) at rest. 
$f_{0}=$ The frequency of the upstream/downstream waves in the stationary laser.

$E_{0}=$ The total energy of the waves comprising the particle (or of the laser's standing waves).

$m_{0}=$ The rest mass of the particle (or mass equivalent of the laser's standing waves).

As there are two waves inside the laser's resonant cavity (upstream \& downstream), the total energy of the waves inside the cavity is:

$$
E_{0}=2 \times\left(e_{0} \times L_{0}\right)
$$

For a laser (or particle) that is moving at Relativistic speed [3], the following equations apply:

Let $e_{u p}=$ energy per unit length of the upstream wave.

Let $e_{\text {down }}=$ energy per unit length of the downstream wave.

The frequency of the upstream wave is:

$$
f_{u p}=f_{0} \times \frac{c}{c-v}
$$

The frequency of the downstream wave is:

$$
f_{\text {down }}=f_{0} \times \frac{c}{c+v}
$$

As the energy per unit length of a wave is proportional to the wave's frequency ( $e \propto f$ ), then Equations (3) and (4) can be rewritten as:

The energy per unit length of the upstream wave is:

$$
e_{u p}=e_{0} \times \frac{c}{c-v}
$$

The energy per unit length of the downstream wave is:

$$
e_{\text {down }}=e_{0} \times \frac{c}{c+v}
$$

So, the total energy of the waves inside the moving laser's cavity (or comprising a moving particle) is expressed:

$$
E_{\text {moving }}=\left(e_{\text {up }} \times L_{\text {moving }}\right)+\left(e_{\text {down }} \times L_{\text {moving }}\right)
$$

where $L_{\text {moving }}$ is the contracted length of the laser cavity due to the laser's Relativistic motion.

$$
L_{\text {moving }}=\frac{L_{0}}{\gamma}
$$

And $\gamma$ is the Lorentz factor used by Special Relativity:

$$
\gamma=\frac{1}{\sqrt{1-\left(\frac{v}{c}\right)^{2}}}
$$

So, by substituting Equations (5), (6) and (8) into (7):

$$
E_{\text {moving }}=\frac{L_{0}}{\gamma}\left(e_{0} \times \frac{c}{c-v}+e_{0} \times \frac{c}{c+v}\right)
$$




$$
\begin{gathered}
E_{\text {moving }}=\frac{L_{0} e_{0}}{\gamma}\left(\frac{c}{c-v}+\frac{c}{c+v}\right) \\
E_{\text {moving }}=\frac{L_{0} e_{0}}{\gamma}\left(\frac{c(c+v)+c(c-v)}{c^{2}-v^{2}}\right) \\
E_{\text {moving }}=\frac{L_{0} e_{0}}{\gamma}\left(\frac{2 c^{2}}{c^{2}-v^{2}}\right) \\
E_{\text {moving }}=\frac{2 L_{0} e_{0}}{\gamma}\left(\frac{c^{2}}{c^{2}-v^{2}}\right) \\
E_{\text {moving }}=\frac{2 L_{0} e_{0}}{\gamma}\left(\frac{1}{\frac{c^{2}-v^{2}}{c^{2}}}\right) \\
E_{\text {moving }}=\frac{2 L_{0} e_{0}}{\gamma}\left(\frac{1}{1-\left(\frac{v}{c}\right)^{2}}\right)
\end{gathered}
$$

Then substituting Equations (9) into (11) gives:

$$
\begin{gathered}
E_{\text {moving }}=\frac{2 L_{0} e_{0}}{\gamma} \times \gamma^{2} \\
E_{\text {moving }}=2 L_{0} e_{0} \gamma
\end{gathered}
$$

Finally, substituting Equations (2) into (12) gives:

$$
E_{\text {moving }}=\gamma \times E_{0}
$$

Then by converting energy into mass equivalent (Equation (1)):

$$
m_{\text {moving }}=\gamma \times m_{0}
$$

This is the mass equivalent of the Relativistic energy increase that occurs to a moving mass $m_{0}$.

\section{Classical Explanation for the Transverse Relativistic Mass Increase}

From Equation (14) we see that the actual Gravitational mass equivalent of the particle's energy when moving at a Relativistic speed is $\gamma$ times its original rest mass $m_{0}$. If a force is applied sideways to the moving particle (in the transverse direction to the particle's direction of motion), there is no increased wave momentum in the particle's standing wave waves in that transverse direction due to the particle's motion, so the inertial mass of the particle will equal its mass due to the equivalent energy it contains (its Gravitational mass). As inertia depends on the rate at which the particle's waves are made to move when a force is applied to them, the wave explanation for the transverse inertia is due to the time dilation of the moving particle's reference frame. All of the waves that comprise the particle are traveling an optical path length that is greater than that of an 
identical stationary particle, by an amount equal to the amount of time dilation in that moving particle (the light clock example often used to explain Time Dilation in Relativity), which is $\gamma$. So, any force propagating through the particle (which it must do in order to make it move) will be traveling a further distance (traveling at the speed of light c) than it would in a stationary particle. So, it will take $\gamma$ times longer to do so, which results in the inertia being $\gamma$ times greater also.

So, the transverse inertial mass $\left(m_{T}\right)$ will also be:

$$
m_{T}=\gamma \times m_{0}
$$

\section{Classical Explanation for the Longitudinal Relativistic Mass Increase}

In the Longitudinal direction (relative to the particle's direction of motion) there is a different inertial mass to the mass of the particle based on that particle's energy content. This is because the waves in the particle's standing wave have been Doppler shifted (due to the particle being in motion) to a higher frequency upstream wave and a lower frequency downstream wave (as we saw in Equations (3) \& (4)). The total momentum is the sum of the upstream and downstream waves which are constantly bouncing backwards and forwards between the particle standing wave's nodes. The nodes have equal but opposite waves impacting them from both sides and so remain stationary, which has the effect of causing each wave to reflect (as would happen to a wave on a rope where the rope is fixed and not allowed to move). At each node, when a reflection occurs, a pressure is exerted and an upstream wave is reflected to become a downstream wave (and vice versa). To calculate the magnitude of the longitudinal inertial mass of a moving particle, based on the knowledge that the particle is comprised of a three-dimensional Electromagnetic standing wave, we must perform the following calculations:

From Equations (5) \& (6) we saw that:

The energy per unit length of the upstream wave is:

$$
e_{u p}=e_{0} \times \frac{c}{c-v}
$$

The energy per unit length of the downstream wave is:

$$
e_{\text {down }}=e_{0} \times \frac{c}{c+v}
$$

So, the total energy per unit length is:

$$
E_{L}=\left(e_{0} \times \frac{c}{c-v}+e_{0} \times \frac{c}{c+v}\right)
$$

The total wave energy reflecting at the nodes each second is:

$$
E_{L} c=\left(e_{0} \times \frac{c^{2}}{c-v}+e_{0} \times \frac{c^{2}}{c+v}\right)
$$

But the relationship between energy and momentum $(p)$ for an electromag- 
netic wave is $E=p c$, so:

$$
p=e_{0}\left(\frac{c}{c-v}+\frac{c}{c+v}\right)
$$

However, as we saw with the transverse inertial mass explanation, time is running slower by a factor of $\gamma$ for the moving particle, which means it takes $\gamma$ times longer for the standing waves in the particle to communicate the momentum change through the particle. This also adds momentum to the particle. So, the total momentum will actually be:

$$
\begin{gathered}
p=\gamma e_{0}\left(\frac{c}{c-v}+\frac{c}{c+v}\right) \\
p=\gamma e_{0}\left(\frac{c(c+v)}{c^{2}-v^{2}}+\frac{c(c-v)}{c^{2}-v^{2}}\right) \\
p=\gamma e_{0}\left(\frac{c^{2}+c v}{c^{2}-v^{2}}+\frac{c^{2}-c v}{c^{2}-v^{2}}\right) \\
p=\gamma e_{0}\left(\frac{2 c^{2}}{c^{2}-v^{2}}\right)=\gamma e_{0} 2 \gamma^{2}=2 e_{0} \gamma^{3}
\end{gathered}
$$

Also, from Equation (2), we saw that the total energy per unit length of stationary particle is:

$$
E_{0}=2 e_{0} c
$$

Thus,

$$
p_{0}=2 e_{0}
$$

So, comparing the longitudinal momentum with the momentum when at rest gives:

$$
\frac{p}{p_{0}}=\frac{2 e_{0} \gamma^{3}}{2 e_{0}}=\gamma^{3}
$$

Therefore [4], the longitudinal mass $\left(m_{L}\right)$ is:

$$
m_{L}=\gamma^{3} m_{0}
$$

\section{Conclusion}

By modeling particles as three-dimensional Electromagnetic standing waves (using only Classical Physics) we are able to understand and visualize how and why the moving particle has different inertial mass in the longitudinal and transverse directions. The derived equations (Equations (15) and (24)) match the currently accepted equations for the inertial mass of a moving body, thus validate this aspect of the model. Some work has been done by others to simplify this directional dependence of inertial mass into a simple mass formula that can be used regardless of the direction of the applied force. This work employs a relativistic subtraction formula to achieve this, but as a result, is not purely Classical in its treatment [5]. 


\section{Conflicts of Interest}

The author declares no conflicts of interest regarding the publication of this paper.

\section{References}

[1] Traill, D.A. (2018) Wave Functions for the Electron and Positron. https://www.researchgate.net/publication/326646134 Wave functions for the elec tron and positron

[2] Lorentz (1899) Simplified Theory of Electrical and Optical in Moving Systems. Proceedings of the Royal Netherlands Academy of Arts and Sciences, 1, 427-442.

[3] Traill, D.A. (2012) A Classical Reconstruction of Relativity. https://www.researchgate.net/publication/343975995 A Classical Reconstruction of Relativity

[4] 195 Longitudinal and Transverse Mass, The Karlsruhe Physics Course. http://www.physikdidaktik.uni-karlsruhe.de/download/195 longitudinal and trans verse mass.pdf

[5] Koczan, G.M. (2020) Defining the Three-Dimensional Acceleration and Inertial Mass Leading to the Simple Form F = MA of the Relativistic Motion Equation. arXiv: 1909v3 [physics-hist-ph] 